\title{
The Design, Operation, and Application of a Dynamic Gastric Model
}

\author{
M. J. S. Wickham ${ }^{1}$, R. M. Faulks'*, J. Mann ${ }^{3}$, and G. Mandalari ${ }^{2}$ \\ ${ }^{1}$ Nutrition Research Department, Leatherhead Food Research, Randalls Road, Leatherhead, \\ Surrey KT22 7RY, UK \\ ${ }^{2}$ Model Gut Platform, Institute of Food Research, Norwich Research Park, Colney, Norwich NR4 7UA, UK \\ ${ }^{3}$ MSD Development Laboratories, Hertford Road, Hoddeston, Hertfordshire EN11 9BU, UK
}

e-mail: martin@pbltechnology

\section{ABSTRACT}

The mechanical functioning of the stomach has been well researched (1). The contractions that mix, break up, and propel the gastric bolus in the main body and antrum have been described in detail and have been partially modeled mathematically. Because the antral forces are particularly important in the mixing and break up of food, they have been measured using manometers, pressure transducers, MRI imaging of agar beads of differing strength (2), and other methods $(3,4)$. The chemical and biochemical environment of the stomach, its acid and digestive enzymes, and their production and activity rates under different conditions have been studied for many years, and reference ranges established mainly for diagnostic purposes. All these areas have been extensively reviewed (5-7).

Despite this understanding of gastric function, many in vitro digestion studies use grossly simplified systems that often include food homogenization, nonphysiological mixing and shear, and unrealistic acid and enzyme concentrations that do not change over time as happens in vivo.

This paper describes the design and operation of a computer-controlled dynamic gastric model (DGM) that was built to investigate the effects of the biochemical and physical processing of foods and oral pharmaceuticals.

Our intention was to draw together the physical and biochemical features of the human stomach with data on gastric residence time and emptying profiles and to design a computer-controlled mechanical stimulation that works in real time with realistic chewed foods or meals and oral pharmaceutical and nutraceutical products.

\section{INTRODUCTION}

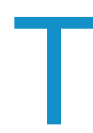

he gastric storage, processing, conditioning, and delivery of ingested foods are vital preliminary elements in the delivery of optimal nutrition and the subsequent maintenance of good health. Consequently, the functions of the stomach have been studied extensively in both health and disease with particular attention given to the gastric luminal environment and the dynamic changes that occur in the fed and fasted states. However, such studies are challenging since invasive interventions in humans for the acquisition of gastric contents or the measurement of the physical activity inevitably lead to difficulties, not the least of which are ethical concerns. For example, only liquid meals can be aspirated, and manometric devices, which are difficult to locate, only give limited information on the complex mixing and shearing of the inhomogeneous gastric food bolus. Remote imaging systems and ingestible sensors have added immensely to our understanding of gastric functioning; however, they are slow and expensive. The use of animal models has questions of suitability and ethics, and current in vitro models are often not realistic of human digestion. Nevertheless, such models can provide insights and comparative data. To overcome these experimental and ethical difficulties, several more sophisticated gastric and intestinal simulators have been developed in recent years and have started to provide useful data in areas such

${ }^{*}$ Corresponding author. as the behavior of functional foods, the survival of probiotics, and the performance of oral drugs $(8,9)$. The development of physical simulators is being paralleled by in silico mathematical modeling of gastric flow patterns, mixing, and shears, which is particularly useful in understanding the behavior of oral drug formulations if dissolution tests fail to predict in vivo behavior.

\section{GENERAL CONSIDERATIONS}

Food in the stomach is usually a masticated mixture of protein, fat, carbohydrate, indigestible components, micronutrients, non-nutrient phytochemicals, microbiota, and water to which has been added a variable amount of saliva containing enzymes, salts, and bacteria. As such, the gastric food bolus is inhomogeneous at many different levels and retains, at least to some extent, the original structures of the foods consumed. Mastication therefore exposes some nutrients to the immediate effects of the buccal and gastric environment while much remains within the structure of the food bolus delivered to the stomach or the food particles themselves. This masticated mixture is loaded into the gastric compartment over time as we eat. The first food bolus swallowed encounters an acidic environment $(\mathrm{pH} \approx 2.0)$ of residual gastric secretions in the lowest part of the stomach, which may vary in volume up to about $50 \mathrm{~mL}$. Subsequently, as further food is added, the bulk pH usually rises to close to that of the food because of its buffering capacity, although the $\mathrm{pH}$ may still be quite 
acidic close to the stomach wall. The transition of gastric contractions from phase I (fasting/resting) to phase II (fed) is rapid so that during meal ingestion, gastric emptying may have already started depending on the time taken to eat and the size, composition, viscosity, temperature, and osmolarity of the meal. Gastric secretions produced from the stomach wall are slowly mixed with the gastric contents through massaging caused by rhythmic gentle peristaltic contractions of the proximal stomach (10). Because the massaging is quite gentle, the gastric secretions may not penetrate to the center of the gastric bolus for up to an hour or more depending on flow characteristics (viscosity and cohesion) of the gastric contents (10) such that pooling of gastric secretions on the top of the meal can be detected in most healthy subjects (11). Fat in the meal, particularly free fat above its melting point, will often separate and float to the top of the gastric contents along with food particles containing air because mixing is insufficient to maintain a homogenous mixture. The gentle massaging and peristalsis in the proximal stomach also selectively pushes the gastric contents into the antrum. This selectivity arises because the part of the gastric bolus in contact with the stomach wall is lubricated by gastric mucus and more hydrated and digested by the gastric secretions. This more fluidized portion, being more mobile (lower viscosity) than the bulk of the gastric contents, is preferentially swept down into the antrum. However, by using computational fluid dynamics modeling, a second process has been proposed. In this case, a contraction in the proximal stomach initiates flow down the center of the gastric bolus, pushing material from the center of the stomach into the antrum and duodenum (12). Yet, it is not clear how important this second model is in the normal healthy stomach, but instances of elevated abdominal pressure (e.g., exertion) could certainly create the conditions needed for this mechanism to operate.

The gentle contractions in the proximal stomach deepen and strengthen as they move down to the antrum. The antrum is therefore a zone of higher shear and mixing than the main body of the stomach. In this region, the contents are more homogenous than in the main body; nevertheless, because of the particulate nature of many chewed food items, homogeneity within the antrum is not likely to be complete unless the luminal content is liquid. The antral contractions push the contents against the closed entrance to the duodenum (pylorus) creating pressure, which dilates the antrum and forces the antral contents to reflux back through the advancing contraction creating the high shear that mixes and breaks down particle size through particleparticle interactions and shear of the viscous matrix. Once the contraction reaches the pylorus, it dies out, the pylorus relaxes, and the more fluid portion of antral contents closest to the pylorus is ejected into the duodenum. This process causes the preferential emptying of liquid and small particles, tending to retain the larger particles for further antral processing. However, much larger particles may pass the pylorus during normal digestion (2), and this allows the use of gastro-protected and enteric-coated oral drug formulations. Larger particles that resist breakup may also be retained until eventually emptied by the action of the so-called housekeeper wave (phase III migrating motor complex). It is quite clear then that the portions of the meal that are emptied from the stomach into the duodenum over time have different compositions, structures, degrees of gastric processing, exposure to gastric acid and enzymes, and mean residence times. A particle of food or a tablet within this dynamic matrix will therefore "see" a complex, changing environment. As yet, it is not possible to provide a mathematical description of the complex gastric processes, but it is possible to construct a physical model that can simulate the key features of the process in terms of time and scale.

\section{CHEMICAL AND BIOCHEMICAL GASTRIC PROCESSES}

The primary purposes of the stomach are to receive and store ingested food and then condition and deliver digesta to the duodenum in a regulated manner. In general, food can be ingested much more rapidly than it can be handled by the upper $\mathrm{Gl}$ tract, and this permits the intake of meals rather than continuous grazing. This means that nutritional requirements can be met in only a few minutes, leaving time for the acquisition of further food or other activities. In adult humans, the gastric capacity is in the range 0.1-4.0 $\mathrm{L}$ (empty-full) although in general we tend not to ingest more than about one liter at a meal; we may well drink volumes in excess of this. As the stomach stretches to accommodate a meal, the production of gastric acid and enzymes increases from basal (fasting) rates to stimulated rates, and this is accompanied by an increase in the rate of flow of gastric juice from around $80 \mathrm{~mL} / \mathrm{h}$ to around $200 \mathrm{~mL} / \mathrm{h}$. The actual changes in rate of production within an individual will vary depending on individual response to the presence of food in the stomach. Generally, however, the composition and rate of addition of gastric secretions are greatest at peak gastric volume and rise and fall as the stomach fills and empties to maintain a crude proportionality.

The conditioning of food in the stomach is achieved through the addition of acid and digestive enzymes secreted by the stomach wall along with mucus. The function of acid is to surface sterilize food, soften food particles, and create a localized low $\mathrm{pH}$ environment that is optimal for the cleavage of pepsinogen and the activity of pepsins (Pepsin A - EC 3.4.23.1 and Pepsin C - EC 3.4.23.3) and gastric lipase (EC 3.1.1.3), the main digestive enzymes of the stomach.

Basal acid production during fasting (around $1-2 \mathrm{mmol} / \mathrm{h}$ ) can rise to around $18 \mathrm{mmol} / \mathrm{h}$ (men) and around $14 \mathrm{mmol} / \mathrm{h}$ (women) at peak stimulation (7). The parietal cells, which produce the acid, are regulated by the vagus nerve and through gastrin secreted in response to the presence of food in the stomach, antrum, and to a lesser extent the small intestine. However, acid production can also be affected by mechanical, chemical, and psychological stimuli (e.g., anxiety, stress). 
In general, the $\mathrm{pH}$ of the gastric contents is around that of the meal consumed and falls to the fasting $\mathrm{pH}(<2.0)$ at the end of gastric emptying. For drinks with little buffering capacity, mixing with the residual gastric secretions in the stomach will effectively bring the $\mathrm{pH}$ down to around the fasting value. The production of gastric acid is subject to control through a pH-regulated feedback loop, while the production of gastric enzymes appears to be stimulated by the presence of food in the stomach.

\section{PHYSICAL GASTRIC PROCESSES}

The simplistic view of physical processing in the stomach is to consider the main body of the stomach as a zone of very gentle mixing and the distal stomach (antrum) as a zone of higher shear and mixing that is mainly responsible for the breakup of food particles before emptying into the duodenum. It is difficult to assess the relative effect of mastication and antral shear on the particle size distribution of the chyme entering the duodenum because of sampling problems in vivo, but there are clearly limits to the forces and work input in the antrum such that many ingested food particles and pharmaceuticals enter the duodenum without further breakdown.

Although it has long been recognized that gastric processing is important, safe noninvasive methods of investigation, particularly magnetic resonance imaging (MRI), have only recently become available. MRI studies have confirmed many previous observations and provided new insights (4), in particular, the slow and gentle mixing in the main body of the stomach that occurs even with slightly viscous fluids has been investigated (10).

The forces in the antrum have also been measured using agar beads of differing strength (2) and more recently by high-resolution, real-time magnetic tracking (13). However, it is not pressure per se that causes particle shearing. Rather it is a combination of two factors, the anisotropic application of force and displacement both axially and radially, coupled with the flow properties of the luminal contents that dictate the flow fields, while the mechanical strength and size of the particles within the fields dictate whether they will undergo particle size reduction through break up, erosion, or attrition.

\section{GASTRIC EMPTYING}

Gastric emptying is a controlled process. Regulation is crucial so that the efficient digestion and absorption of available nutrients can occur without overwhelming the capacity of the digestive enzymes, the ability of the small intestine to absorb the low molecular weight products of digestion, and the maintenance of metabolic control. How fast the stomach empties appears to depend on the energy density of the food, the sources of the energy, viscosity of the gastric contents, and gastric distension. Non-nutrient drinks and meals tend to empty more rapidly. Water may empty at about $20 \mathrm{~mL} / \mathrm{min}$, easily digested carbohydrates at $10 \mathrm{kcal} / \mathrm{min}$, and lipid emulsions at 2-3 $\mathrm{kcal} / \mathrm{min}$. The meal composition and volume therefore have an impact on the total gastric residence time. Regulation is mainly through the gut sensing the presence of absorbable nutrients and providing the feedback that controls delivery from the stomach and mass transport along the small intestine (intestinal motility). There are many signaling systems; some appear to be driven by physical factors (gastric distension, meal temperature, osmolarity) and some are driven in response to the presence of nutrients or the site at which they are absorbed. For example, cholecystokinin (CCK) produced in the duodenal and jejunal I cells increases satiety and stimulates gall bladder contraction, ghrelin produced in the stomach and pancreas stimulates hunger, and leptin produced in adipose tissue suppresses hunger. Gastrin produced by antrum and stomach $\mathrm{G}$ cells controls gastric acid production, and peptide YY (PYY) produced by the distal ileum and proximal colon reduces the rate of gastric emptying and lowers food intake.

At present, there is no clear model of the interactions of the various stimulatory and inhibitory effects of the enteroendocrine systems on gastric processing, emptying, satiety, hunger, and the subsequent effects on plasmaloading profiles, body mass, and health. However, as information on the interactions is uncovered, the data can be integrated into model control systems and ultimately used to fine-tune the current relatively crude energybased model of gastric emptying.

\section{MODEL SPECIFICATIONS}

A realistic model should replicate not only the dynamic chemical, biochemical, and physical processes that occur during the processing of a meal in vivo, it must also work in real time with real foods, meals, or pharmaceuticals. We have constructed such a model (Figure 1) in so far as we have been able to replicate those processes that are essential to gastric processing (14). In an ideal world, a physical model would be a full simulation, but this is not possible. The specification is therefore divided into what the current dynamic gastric model (DGM), developed at the Institute of Food Research, does and a wish list for future iterations.

\section{GENERAL SPECIFICATION Model Functions}

- Is capable of processing drinks, normal-size chewed meals, and oral pharmaceuticals.

- Operates at $37^{\circ} \mathrm{C}$ with heat transfer rates similar to those in vivo.

- Operates and processes meals in real time.

- Creates gentle cyclical $(0.05 \mathrm{~Hz})$ mixing in the main body of the stomach.

- Has physiological rates of acid, salts, and enzyme addition around the gastric bolus.

- Controls and measures acid addition that results in flow rates that change automatically depending on meal and luminal contents. 
- Controls and measures enzyme additions that result in flow rates that automatically change depending on meal and luminal contents.

- Creates antral shear rates and shear forces as in vivo calibrated with agar beads (15).

- Avoids inappropriate crushing/shearing.

- Has antral frequency $(0.05 \mathrm{~Hz})$ and waveform as in vivo phase Il contractions.

- Has preferential antral sieving.

- Controls and measures the rate of emptying.

- Empties in a series of pulses over the residence time of the meal.

\section{Model Operation}

- Loads chewed meal/drink and sets initial flow rates of gastric secretions.

- Is autonomous once started.

- Date stamps all variables logged over time for quality control purposes.

- Does not contaminate samples through corrosion or absorption.

- Is easily dismantled and reassembled for cleaning and may be chemically or thermally sterilized.

- Permits sampling from main body and antrum.

\section{Wish List}

- The whole model is transparent.

- Real-time, online chemical and physical analysis of the digestion process.

- Integration of real time digesta analysis with gastric emptying.

An additional point about the model is that although the initial parameters are programmed, outputs in terms of process time and volumes vary because of the inhomogeneity of the meal, as might be expected in vivo.

\section{Construction}

The main body of the stomach is a cone-shaped elastic membrane. The membrane stretches when the meal is loaded; it also allows rapid heat transfer from and to the surrounding water bath and the massaging of the contents by the application of cyclically (sine wave) variable external pressure. The degree of distortion of the main body is dependent on the radius of curvature such that the greatest distortion occurs at the top of the flexible section. The addition of acid and enzymes occurs through the perforated hoop lying on the wall of the "stomach," and the cyclical compression and relaxation of the stomach draws the added acid and enzyme over the inside surface of the main body. The $\mathrm{pH}$ in the main body is monitored by a series of flexible nasogastric $\mathrm{pH}$ electrodes that provide the feedback to control acid-addition rate. The geometry favors the induction of the most fluid part of the gastric bolus into the antrum by the movement of the piston. The contents of the antrum are then sheared and mixed by the cyclical movement of the barrel, to which an elastic annulus

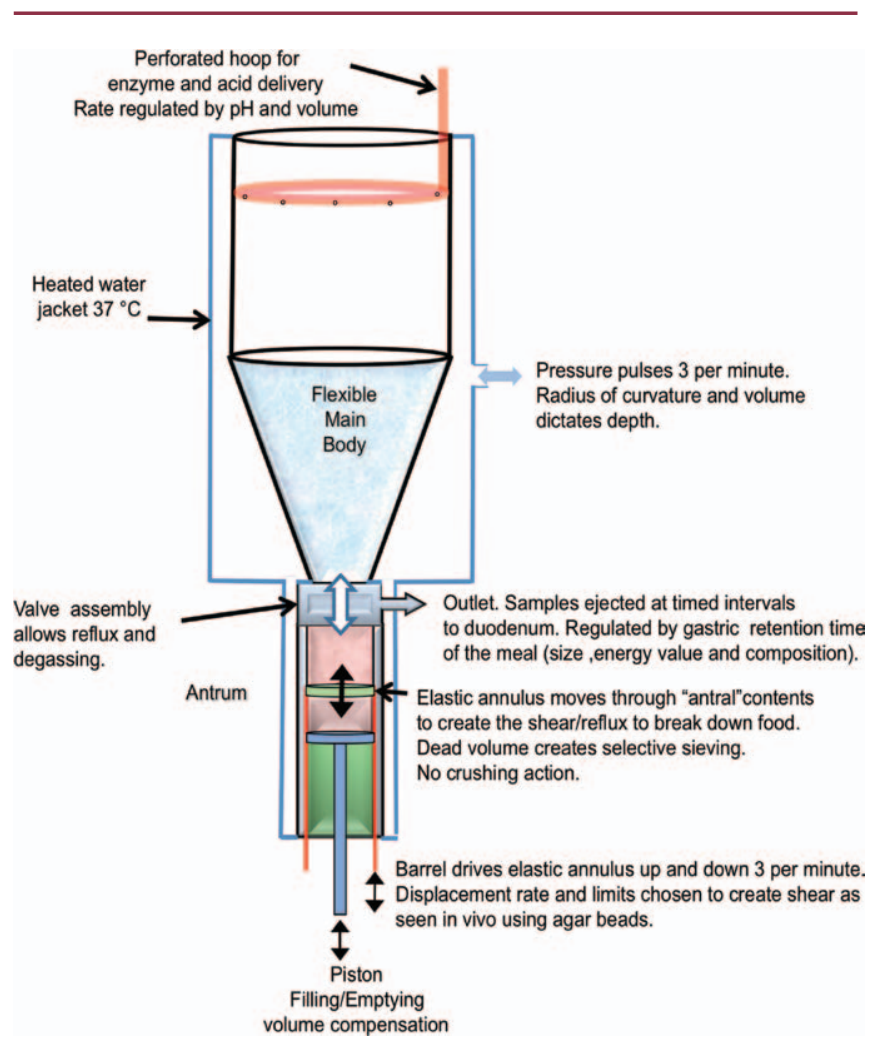

Figure 1. DGM schematic (not to scale). The unit replicates the internal volumes of the average human stomach, and operates in real time and within physiological references ranges (14).

is attached, while volume compensation is permitted by minor adjustments of the piston position if required. The meal in the antrum therefore fluxes back and forth (axial forces) through the elastic annulus (radial forces) simulating the squeezing, propulsion, retropulsion, attrition, erosion, and shearing as in antral processing in vivo. The barrel and piston assembly is set to provide a dead volume at the end of each stroke to avoid artificial mechanical crushing, and the sequence of valve operations is such that the valve between the main body and the antrum is open except when the antrum is emptying. This permits degassing and reflux between the main body and the antrum while allowing accurate control of the volumes processed. The whole volume of the antrum is not swept at each emptying cycle so that a portion is retained and is mixed with the next portion to be drawn in. This creates a dead volume for the retention of the larger particles, which are then subjected to further antral processing. Once the stomach is empty, the model can perform a "housekeeper" contraction (phase III, migrating motor complex) to ensure that the whole meal is emptied.

\section{Operation}

The system is programmed by using an in silico prediction, derived from in vivo data, of the total gastric retention time based on the meal size, energy density, nutritional composition and viscosity, and the sampling size and frequency desired. Programs can be saved and recalled as 
needed. The initial rates of addition of acid and enzyme are set within the normal physiological range according to the meal size but can be varied to account for some disease states or the effect of drugs, for example, proton pump inhibitors. Contraction cycles are set to three per minute to simulate phase II contractions seen in vivo. The meal may be loaded in real time using "chew and spit," or the chewed meal may be accumulated and added as a single bolus.

Alternatively, to avoid the variations among individuals, the meal may be subjected to a consistent blending procedure during which simulated saliva may be added. The amount of simulated saliva required can be estimated from undertaking a chew and spit of an identical meal and recording the weights before and after chewing. Oral pharmaceuticals may be added at any time before, during, or after the "ingestion" of the test meal with or without a drink. Should the model need to be kept running after the meal has been emptied, for example, to assess gastric retention of a drug formulation, a fasting program with basal gastric secretions can be set to follow on including intermittent phase III contractions at preset intervals. Additional sequences may also be added as needed to simulate processing of multiple meals. Once the program has been initiated, there is no further operator input other than to collect the samples as they are ejected from the antrum. All variables are logged into a date-stamped, read-only file.

Data that are acquired:

- Program name, date, operator, and programmable variables.

- Gastric pH-v-time.

- Acid addition-v-time and total ( $\mathrm{mL}$ or $\mathrm{mmol}$ ).

- Enzyme addition-v-time and totals (volume or units).

- Temperature-v-time.

- Gastric retention time ( $t_{1 / 2}$ or total).

Data that can be obtained:

- Emptying profile (mass-v-time) by weighing or analyzing each antral sample.

- Emptying profile (mass-v-time) of components (e.g., pharmaceuticals).

- Antral pH (as each sample is emptied).

- Enzyme digestion of gastric contents over time by analysis.

- Particle size reduction.

- Behavior of gastro-retentive/-resistant formulations.

- Dilution by gastric secretions.

- Solid/dissolved ratios of components.

- Mass transfer of components between phases.

- Rupture time of capsules and delivery profile of active to duodenum.

- Emulsification of lipid in stomach/antrum.

- Phase separation (lipid and low/high density components).

- Gel formation and effects of antral shear.

- Rate of creation of absorbable forms (nutrients, drugs).

- Video footage of stomach contents from the surface.

\section{Variants}

Because both the physical and biochemical parameter sets are independently programmable, adjustments may be made for various pathological conditions and drug effects where there is reliable data. The model may also be rescaled to provide a pediatric model at nine months and beyond.

\section{Application}

Although the DGM was originally constructed to assess the impact of the first stages of digestion on the bioaccessibility and delivery profile of nutrients to the duodenum to understand the timing and magnitude of plasma excursions, the same approach can be used for oral drug delivery. The disintegration, dissolution, and delivery to the duodenum are key features in determining the rate of exposure of the absorption surface to potentially absorbable species. Such data should allow in vitro-in vivo correlations (IVIVC) irrespective of the permeability of the compound and are only constrained if the absorption kinetics become saturated (nonlinear).

\section{Case Studies}

The following studies have been published with the permission of the companies involved or are published validation studies carried out independently by the Model Gut Group.

\section{Gastric Behavior of Different Capsule Shell Materials}

In this study (16), different capsule shell materials filled with paracetamol were tested in both the DGM and a standard USP dissolution Apparatus 1, and the delivery of paracetamol was measured in both the fed and fasted states. Of the capsule shell types, hydroxypropyl methylcellulose with carageenan (HPMC-C) and hard gelatin (HG) showed similar rupture times in Apparatus 1 and in the fasted DGM. However, hydroxypropyl methylcellulose with gellan (HPMC-G) capsules were much slower to rupture and deliver their contents in Apparatus 1 but only lagged HPMC-C and HG capsules by a few minutes in the fasted DGM. The fasted-state rupture times of all the capsule types and gastric emptying profiles of paracetamol measured by the DGM agree with those assessed by gamma scintigraphy and plasma profiling in humans. All three capsule types behaved similarly in the fed state where the lag before the paracetamol empted into the duodenum, caused by the presence of food, nullified the difference in performance of the capsule shell (Figure 2).

\section{Bioequivalence of Complex Dosage Forms}

In this study (17), the intention was to create a bilayer tablet containing multiple APIs in an immediate-release (IR) layer and a nondisintegrating controlled-release layer, then match the in vivo performance of a reference product of the drug contained within the immediate-release layer. In USP dissolution Apparatus 2 (Figure $3 \mathrm{~A}$ ), the immediate-release layer behaved similarly when compressed as a single- 

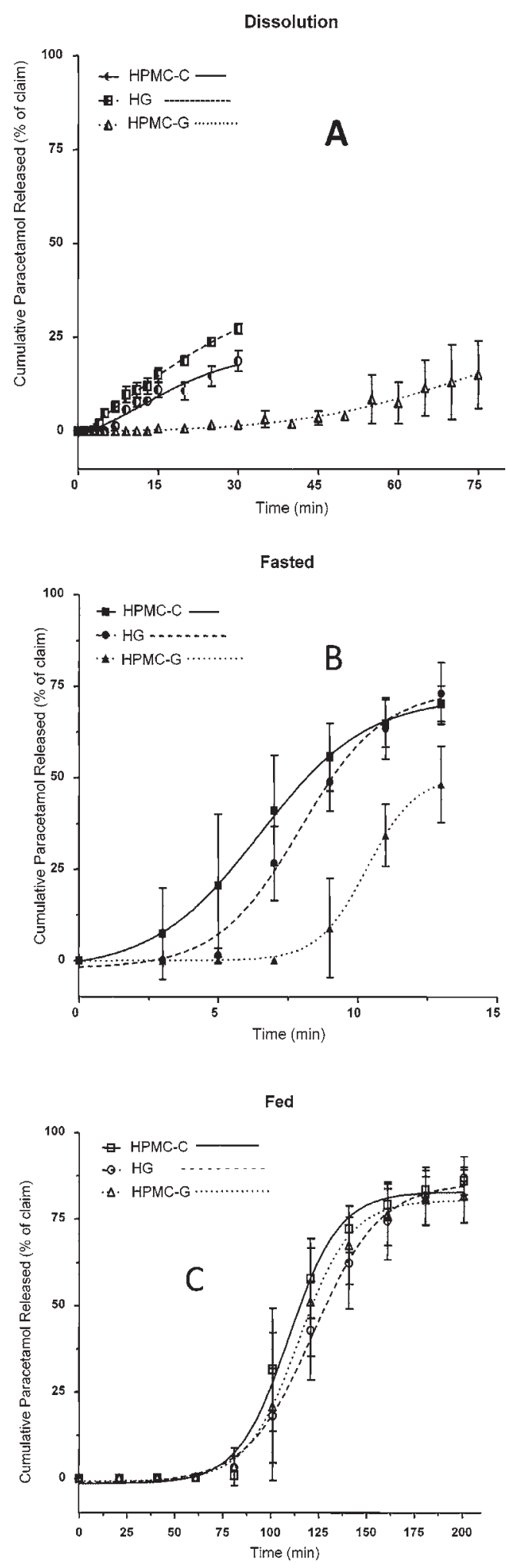

Figure 2. Capsule shell performance. Cumulative paracetamol \% release (of label claim) from three capsule types: hydroxypropyl methylcellulose (HPMC) capsules with carageenan as gelling agent (HPMC-C), HPMC capsules with gellan gum as gelling agent (HPMC-G), and hard gelatin (HG). The capsules were tested in (A) USP Apparatus 1 containing AJPwp, (B) DGM fasted state, and (C) DGM fed state. (Reproduced with permission from ref 16. Copyright 2011 Elsevier B.V.)

entity tablet or a bilayer formulation. In vivo equivalence between the single-entity tablet and the reference product had already been demonstrated, so the delay in Apparatus 2 dissolution was not considered significant in vivo. However, when the bilayer tablet was run in vivo, it was not equivalent to the reference product with reductions in area under the curve $(A \cup C)$ and $C_{\max }$ observed. Testing in the DGM identified that the presence of the controlled-release layer resulted in enough of a gastric retention to delay release when compared with the reference product and the single-entity tablet (Figure 3B). The DGM was then used to screen two reformulations such that the immediate-release layers would delaminate from the controlled-release layer faster and not be gastric-retained. Both reformulations were tested in vivo, and improvements in $A U C$ and $C_{\max }$ trended with the observations made in the DGM.

\section{Self-Emulsifying Drug Delivery Systems}

One of the important points about a self-emulsifying drug delivery system (SEDDS) is that the emulsion created in the stomach should have the desired drug delivery profile, and this depends critically on the size of the emulsion droplets (surface area) and the quality of the interface that controls mass transfer and lipolysis (18). Surface area and interface quality depend on the SEDDS formulation, composition of the environment (fed or fasting) in the stomach, the rate of shear in the antrum (mainly), and the drug loading. Comparisons of data from the volumetric flask, Apparatus 2, and the DGM indicate that the presence of the drug ( $6 \%$ ibuprofen) reduced droplet size in all cases, but it was also observed that the DGM created droplets that were much smaller (5-10') than the other two methods. These smaller droplets were also stable in the simulated gastric environment. This indicates that although the SEDDS did deliver a population of droplets, it was the antral shear that dominated, eliminating the differences in the droplet size profile seen in the drug-loaded and placebo formulations.

\section{Adalat-Coral Comparison for Dose Dumping}

The novel formulation of current drugs to provide outcomes that are more efficacious is an important element of pharmaceutical research. However, the use of the various USP methods and their variants may give rise to the impression that two products are bioequivalent, but this is not borne out when tested in vivo. When tested under fasting conditions in the DGM, the controlled-release erodible tablet (Coral) clearly showed an early burst of release, while the osmotic push/pull formulation (Adalat) showed some delay.

\section{Alcohol Effects}

Alcohol interaction with oral drugs is difficult to assess in human volunteers, and the use of realistic models offers an opportunity to study the relative effects of formulation behavior and drug dissolution on delivery kinetics. In this 

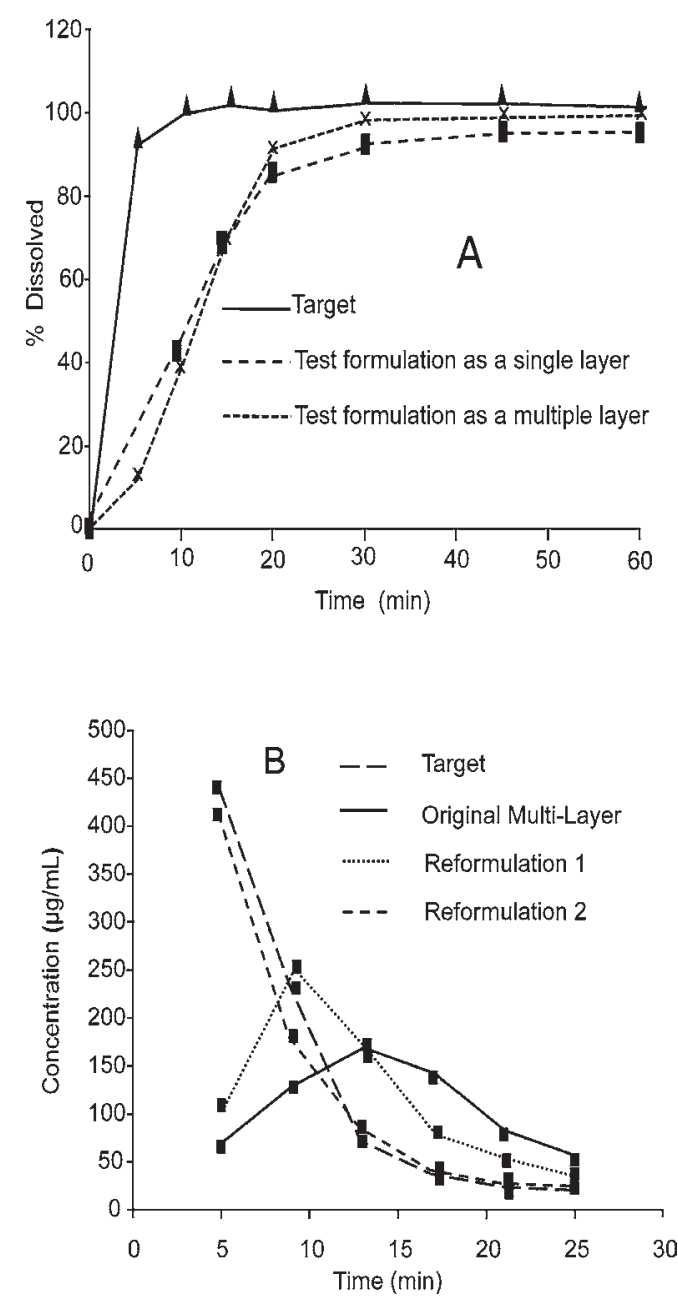

Figure 3. (A) Dissolution in USP Apparatus 1 as a single tablet and as a multilayer tablet. (B) Delivery profile of dissolved drug delivered from the DGM in the fasted state with different formulations. (Reproduced with permission from ref 17. Copyright 2012 Dissolution Technologies, Inc.)

study (19), we were able to build IVIVR/IVIVC for IR capsules containing the poorly water-soluble drug nifedipine when ethanol is coadministered. With the use of Apparatus 1 and the DGM, it was possible to match the in vivo behavior of the formulation in the presence of ethanol. Specifically, using Apparatus 1 and the DGM for alcohol studies may offer meaningful insights on the effect of the coadministration of alcohol with the formulation under investigation.

\section{Allergenic Proteins}

In sensitized (allergic) individuals, the presence of the allergenic protein in foods can cause a reaction ranging from mild discomfort to anaphylaxis. In the more serious cases, urgent medical intervention may be needed. However, it is not clear how long the allergenic stimulus is active in the upper $\mathrm{Gl}$ tract, and this is important in determining the duration of medical intervention. We have carried out digestion studies on peanuts and milk in healthy volunteers using nasogastric and nasoduodenal aspiration and used the DGM to compare the rates of disappearance of the recognized allergenic proteins and the fate of the peptides produced (20). We have demonstrated that the material emptied from the stomach in vivo is very closely matched in both the degree of disappearance of the native proteins and the appearance and profile of the hydrolysis products to that obtained from the DGM. More studies have been carried out to investigate the rate and kinetics of almond protein digestion in the upper $\mathrm{Gl}$ tract and the role played by the food matrix in regulating protein release. We have demonstrated that prunin, the major allergenic protein in almonds, is quickly degraded by pepsin in the gastric compartment when almond flour is ingested with water, whereas much slower release kinetics is observed when almond flour is incorporated in a chocolate dessert matrix or in a Victoria sponge (21). This data has been validated with immunoreactivity experiments using almond-specific antibodies.

\section{Glycemic Index}

Currently glycemic index is a measure of the digestibility of starch-containing foods based on the plasma glucose excursions they provoke after correction for "available-starch" load. However, the plasma response is not just a measure of starch digestion; it also includes the rate of loading of the upper small intestine-the rate of gastric emptying taking into account both volume and concentration of starch substrate. We have used the DGM to investigate the "duodenal" loading profile together with simulated duodenal digestion to produce curves of the "rate of production of absorbable species" and have compared this with the timing and plasma excursions in vivo. The data indicate a good correlation with the GI AUC over the same time scales (22).

\section{Probiotic Survival}

The consumption of foods containing large numbers (i.e., $10^{10}-10^{12}$ viable cells $/ \mathrm{g}$ ) of probiotic "friendly" bacteria, mainly Lactbacillus or Bifidobacteria spp., to improve general health and that of the large bowel requires that the bacteria reach and colonize the colon in sufficient numbers. The upper Gl tract, and in particular the stomach, is designed to kill or disable ingested microorganisms. The DGM coupled with a static duodenal model has been used to assess the survival of various organisms in different food systems (23). We have demonstrated that there is a correlation between probiotic survival and $\mathrm{pH}$ decrease in the stomach and that the food vehicle plays an important role in determining lactobacilli survival in the upper Gl tract (24).

\section{Additional Data}

Because each sample emptied from the antrum is collected separately, each can be transferred wholly or in part to a static duodenal digestion model. This can be used to assess the impact of $\mathrm{pH}$ change and the addition of 
pancreatic enzymes and bile salts. The duodenal process can be used, for example, to predict glycemic index, the persistence of allergenic proteins, the survival of probiotics, the liberation of encapsulated materials, the integrity of oral doses, and duodenal loading curves.

\section{CONCLUSION}

The Dynamic Gastric Model can provide very useful data on the gastric behavior of foods, oral pharmaceutical formulations, and food-drug interactions that have not been easily assessable by other means. Data from the DGM has already been used to help understand gastric behavior and to guide formulation to meet objectives. The full utility of the model is still being explored, particularly with regard to pediatric and gastrointestinal disease states.

\section{REFERENCES}

1. Lentle, R. G.; Janssen, P. W. M. The Physical Processes of Digestion; Springer: New York, 2011.

2. Marciani, L.; Gowland, P. A.; Fillery-Travis, A.; Manoj, P.; Wright, J.; Smith, A.; Young, P.; Moore, R.; Spiller, R. C. Assessment of antral grinding of a model solid meal with echo-planar imaging. Am. J. Physiol. Gastrointest. Liver Physiol. 2001, 280 (5), G844-G849.

3. Szarka, L. A.; Camilliri, M. Methods for measurement of gastric motility. Am. J. Physiol. Gastrointest. Liver Physiol. 2009, 296 (3), G461-G475.

4. Schwizer, W.; Steingoetter, A.; Fox, M. Magnetic resonance imaging for the assessment of gastrointestinal function. Scand. J. Gastroentero. 2006, 41, 1245-1260.

5. Kong, F.; Singh, R. P. Disintegration of Solid Foods in the Human Stomach. J. Food. Sci. 2008, 73 (5), R67-R80.

6. Kong, F.; Singh, R. P. A Human Gastric Simulator (HGS) to Study Food Digestion in Human Stomach. J. Food. Sci. 2010, 75 (9), E627-E635.

7. Lentner, C., Ed. Geigy Scientific Tables: Units of Measurement, Body Fluids, Composition of the Body, Nutrition, 8th ed.; Ciba-Geigy Ltd.: Basel, Switzerland, 1981; Vol. 1.

8. Blanquet, S.; Zeijdner, E.; Beyssac, E.; Meunier, J. P.; Denis, S.; Hayenaar, R.; Alric, M. A dynamic artificial gastrointestinal system for studying the behavior of orally administered drug dosage forms under various physiological conditions. Pharm. Res. 2004, 21 (4), 585-591.

9. Sumeri, I.; Arike, L.; Adamberg, K.; Paalme, T. Single bioreactor gastrointestinal tract simulator for study of survival of probiotic bacteria. Appl. Microbiol. Biotechnol. 2008, 80 (2), 317-324.

10. Marciani, L.; Gowland, P. A.; Spiller, R. C.; Manoj, P.; Moore, R.; Young, P.; Fillery-Travis, A. Effect of meal viscosity and nutrients on satiety, intragastric dilution and emptying assessed by MRI. Am. J. Physiol. Gastrointest. Liver Physiol. 2001, 280 (6), G1227-G1233,

11. Holloway, R. H.; Sifrim, D. A. The acid pocket and its relevance to reflux disease. Gut 2008, 57, 285-286.
12. Pal, A.; Brasseur, J. G.; Abrahmsson, B. A stomach road or "Magenstrasse" for gastric emptying. J. Biomech. 2007, 40, 1202-1210.

13. Laulicht, B.; Tripathi, A.; Schlageter, V.; Kucera, P.; Mathiowitz, E. Understanding gastric forces calculated from high-resolution pill tracking. Proc. Natl. Acad. Sci. U.S.A. 2010, 107 (18), 8201-8206.

14. Wickham, M.; Faulks, R. Dynamic Gastric Model. U.S. Patent 8,092,222, January 10, 2012.

15. Vardakou, M.; Mercuri, A.; Barker, S. A.; Craig, D. Q. M.; Faulks, R.; Wickham, S. J. Achieving Antral Grinding Forces in Biorelevant In Vitro Models: Comparing the USP Dissolution Apparatus II and the Dynamic Gastric Model with Human In Vivo Data. AAPS PharmSciTech 2011, 12 (2), 620-626.

16. Vardakou, M.; Mercuri, A.; Naylor, T. A.; Rizzo, D.; Butler, J. M.; Connolly, P. C.; Wickham, M. S. J.; Faulks, R. M. Predicting the human in vivo performance of different oral capsule shell types using a novel in vitro dynamic gastric model. Int. J. Pharm. 2011419 (1-2), 192-199.

17. Mann, J. C.; Pygall, S. R. A formulation case study comparing the Dynamic Gastric Model (DGM) with conventional dissolution methods. Dissolution Technol., in press.

18. Mercuri, A.; Passalacqua, A.; Wickham, M. S.; Faulks, R. M.; Craig, D. Q. M.; Barker, S. A. The Effect of Composition and Gastric Conditions on the SelfEmulsification Process of Ibuprofen-Loaded SelfEmulsifying Drug Delivery Systems: A Microscopic and Dynamic Gastric Model Study. Pharm Res. 2011, 28 (7), 1540-1551.

19. Mercuri, A.; Faulks, R.; Barker, S.; Huatan, H. Institute of Food Research, Colney, Norwich, UK and University of East Anglia, Norwich, UK. Effect of the intake of an alcoholic drink on IR capsules in the gastric compartment. Unpublished work, 2010.

20. Mandalari, G.; Faulks, R.; Mills, C. The generation of allergenic peptide species during digestion of milk and peanut proteins. Allergy, to be submitted for publication.

21. Mandalari, G.; Faulks, R. Institute of Food Research, Colney, Norwich, UK. Unpublished work, 2011.

22. Ballance, S.; Lea, P.; Sahlstrøm, S.; Nagy, N. E.; Anderson, P. V.; Dessev, T.; Vardakou, M.; Faulks, R. Evaluation of gastric processing and duodenal digestion of starch in six cereal meals using an adult fasted dynamic gastric model. Eur. J. Nutr., in press.

23. Pitino, I.; Randazzo, C. L.; Mandalari, G.; Lo Curto, A.; Faulks, R. M.; Le Marc, Y.; Bisignano, C.; Caggia, C.; Wickham, M. S. Survival of Lactobacillus rhamnosus strains in the upper gastrointestinal tract. Food Microbiol. 2010, 27 (8), 1121-1127.

24. Lo Curto, A.; Pitino, I.; Mandalari, G.; Dainty, J. R.; Faulks, R. M.; Wickham, M. S. J. Survival of probiotic lactobacilli in the upper gastrointestinal tract using an in vitro gastric model of digestion. Food Microbiol. 2011, 28 (7), 1359-1366. 